\title{
First-principles investigations of the dielectric properties of polypropylene/metal-oxide interfaces
}

\author{
Liping Yu, V. Ranjan, M. Buongiorno Nardelli, and J. Bernholc \\ Center for High Performance Simulation and Department of Physics, North Carolina State University, \\ Raleigh, North Carolina 27695, USA
}

(Received 4 August 2009; published 30 October 2009)

\begin{abstract}
Nanoscale-resolved dielectric properties of polypropylene/metal-oxide (alumina, $\mathrm{PbTiO}_{3}$ ) interfaces and of the corresponding surfaces are investigated via first-principles calculations. In order to ascertain the locality of the atomically resolved permittivity profiles, we propose a simple procedure to directly evaluate the real-space decay length of nonlocal effects in the dielectric susceptibility. Based on this decay length, the microscopic dielectric response is derived by using a convolution of rectangular and Gaussian filters as the averaging weight function. This procedure converges quickly to the bulk values in slabs of only moderate thicknesses, while providing atomic-layer-resolved permittivity profiles even in the presence of significant relaxations and surface structure. Our results show that (i) the surface-induced and interface-induced modifications to the dielectric permittivity in polymer/metal-oxide composites are localized to only a few atomic layers; (ii) the interface effects are mainly confined to the metal-oxide side; and (iii) metal-oxide particles larger than a few nanometers should retain the average macroscopic value of their bulk dielectric permittivities.
\end{abstract}

DOI: 10.1103/PhysRevB.80.165432 PACS number(s): 77.55.+f, 73.61. $-\mathrm{r}$, 73.20. $-\mathrm{r}$, 71.15.Mb

\section{INTRODUCTION}

Recently, the growing need for high-power-density capacitors and electrical energy storage devices has stimulated intensive research efforts on polymer-ceramic nanocomposites, in which metal-oxide nanoparticles are incorporated into a polymer matrix. ${ }^{1-5}$ Polymers such as polypropylene generally have high dielectric strength, good flexibility, and easy processability at low temperatures, but also have a low dielectric constant, which limits their abilities to store energy. On the other hand, metal oxides such as ferroelectric $\mathrm{PbTiO}_{3}$ possess very high dielectric permittivity, but are brittle, have low dielectric breakdown fields, and need high-temperature sintering. When these two materials are integrated into a nanocomposite, they offer the possibility of achieving high energy and power densities, potentially enabling a broad range of applications in aerospace, automotive, and power industries. ${ }^{3}$

The combination of the high- $k$ metal-oxide nanoparticles with polymers usually results in an effective composite dielectric permittivity, $\varepsilon$, higher than the pure polymer matrix and lower than the nanoparticles. ${ }^{1}$ The general understanding of the role of interface effects is currently still limited to a qualitative level, ${ }^{3,6}$ and is mainly based on classical approximations such as various mixing models, ${ }^{7}$ and the classical effective-medium method. ${ }^{8}$ However, as the incorporated particles shrink to nanometer sizes, their dielectric properties may become quite different from the corresponding bulk materials. The large interfacial area also leads to significant interfacial volume, which may affect the properties of both the nanoparticles and the polymer. These effects are investigated at the microscopic, quantum-mechanical level in this work.

Recently, Giustino and Pasquarello ${ }^{9}$ developed a firstprinciples theory for investigating atomic-scale permittivity profiles across interfaces between insulators. It has been successfully applied to several systems such as $\mathrm{Si} / \mathrm{SiO}_{2},{ }^{9}$ $\mathrm{SiO}_{2} / \mathrm{C}_{12} \mathrm{H}_{25}$ chain, ${ }^{10}$ and metal $/ \mathrm{SrTiO}_{3}$ interfaces. ${ }^{11}$ In this theory, a local susceptibility is introduced to describe varia- tions in the dielectric response over length scales on the order of interatomic distances. It assumes that the nonlocality of the microscopic susceptibility tensor occurs at smaller distances. In fact, this assumption may not always be true. For a homogeneous system, as already shown by Giustino and Pasquarello, ${ }^{9}$ the typical real-space decay length $\xi$ of the nonlocal effects in the dielectric function is larger than the spreads of maximally localized Wannier functions. ${ }^{12}$ The latter are usually on the order of interatomic distances. ${ }^{13}$ In particular, for a system where the lattice contribution dominates the low-frequency dielectric response, we will show that the decay length $\xi$ can be much larger than an interatomic distance. Furthermore, $\xi$ may differ substantially between systems. Therefore, to properly average and obtain nanoscale-resolved, local and physically meaningful permittivity profiles, one needs to determine the decay length $\xi$ of the nonlocal susceptibility for each specific system under investigation.

In this work, we will evaluate $\xi$ directly by calculating the spatial spread of the change density induced by slightly displacing each nonequivalent atom from its equilibrium position. Using the calculated $\xi$, we introduce a modified averaging model that ensures the locality of the dielectric permittivity profile extracted from $a b$ initio calculations, while efficiently filtering out the physically uninteresting oscillations due to the lattice periodicity. The resulting profiles are flat in the bulklike region and atomically smooth in the interface and surface regions, while still providing nanoscale resolution.

The main goal of the present work is to investigate the local dielectric permittivity profiles of polymer/metal-oxide nanocomposites, which are being considered for high-powerdensity capacitors. These composites would combine the advantages of polymers, which are light, flexible and can withstand high electric fields, but have low dielectric constants, with those of oxides, which are usually brittle and heavy, with low breakdown fields, but their dielectric constants are usually much larger. A composite material, consisting of oxide particles embedded in a polymeric matrix, would avoid 
the brittleness of the bulk oxides while still taking advantage of their large dielectric constants. We choose isotactic polypropylene (iPP) as the host polymer matrix, because it is one of the most commonly used capacitor dielectrics. For metal oxides, we select alumina $\left(\alpha-\mathrm{Al}_{2} \mathrm{O}_{3}\right)$ and tetragonal $\mathrm{PbTiO}_{3}$, representing two categories of materials of current interest, a nonferroelectric and a high- $k$ ferroelectric. We calculate the optical and static dielectric permittivity profiles across both single bare slabs of these materials and of their interfaces with iPP, with the goal of understanding the nanoscale variation in surface-induced and interface-induced changes to the local dielectric properties. These quantities, which may be very hard to measure, are needed for accurate mesoscopic modeling of dielectric properties of the complex nanocomposites. ${ }^{14}$

The rest part of this paper is organized as follows. In Sec. II, we briefly review the theory of local dielectric permittivity theory, focusing on aspects important to nanoscale averaging. Section III describes the procedures used to evaluate the decay length $\xi$ of the microscopic susceptibility and to obtain the permittivity profiles. Section IV, Results and Discussion, introduces and discusses the dielectric permittivity profiles across metal-oxide and iPP slabs and their interfaces. The summary and conclusions of the paper are presented in Sec. V.

\section{THEORY OF LOCAL DIELECTRIC PERMITTIVITY}

In the following we assume that the interface is oriented along the $z$ axis and each material is periodic in the plane parallel to the interface, referred as the $x y$ plane. Focusing on the dielectric permittivity across the surface or interface, we eliminate the in-plane dependence by taking a planar average of the corresponding microscopic quantity, e.g., for the charge density we define $\bar{\rho}(z)=\frac{1}{S} \int_{S} \rho(\vec{r}) d x d y$, where $S$ is the area of the $x y$ cross section of the unit cell. This transverse averaging has no effect on the Poisson equation. ${ }^{15}$ Starting from the plane-averaged quantities and following Giustino and Pasquarello, ${ }^{9}$ we derive elements of the local dielectric permittivity theory, focusing on the connection between microscopic and macroscopic quantities. At the microscopic scale in the linear regime, the susceptibility $\chi\left(z, z^{\prime}\right)$ and permittivity $\varepsilon\left(z, z^{\prime}\right)$ are defined through

$$
\bar{D}(z)=\int \varepsilon\left(z, z^{\prime}\right) \bar{E}\left(z^{\prime}\right) d z^{\prime},
$$

and

$$
\bar{P}(z)=\int \chi\left(z, z^{\prime}\right) \bar{E}\left(z^{\prime}\right) d z^{\prime},
$$

which are related to each other by

$$
\varepsilon\left(z, z^{\prime}\right)=\delta\left(z-z^{\prime}\right)+4 \pi \chi\left(z, z^{\prime}\right) .
$$

In Eqs. (1) and (2), $\bar{P}(z)$ and $\bar{E}(z)$ correspond to the induced polarization $^{16}$ and the change in the total electric field due to an applied external field. They can be obtained from the induced charge density using

$$
\frac{d}{d z} \bar{E}(z)=4 \pi \bar{\rho}_{\text {ind }}(z)
$$

and

$$
\frac{d}{d z} \bar{P}(z)=-\bar{\rho}_{\text {ind }}(z) .
$$

The microscopic electric displacement $\bar{D}(z)$ in Eq. (1) is defined as $\bar{D}(z)=\bar{E}(z)+4 \pi \bar{P}(z)$ and satisfies

$$
\frac{d}{d z} \bar{D}(z)=0
$$

which shows that $\bar{D}(z)$ is a constant.

The microscopic permittivity $\varepsilon\left(z, z^{\prime}\right)$ and susceptibility $\chi\left(z, z^{\prime}\right)$ as defined in Eqs. (1) and (2) are nonlocal and usually vary very rapidly over interatomic distances. In order to obtain measurable local dielectric properties from the microscopic quantum-mechanical results, a spatial-averaging procedure needs to be adopted. Denoting the averaging distance as $r_{0}$, it must be significantly larger than the interatomic distance, so that the dielectric-response function can be considered local. For nanostructures, in order to obtain a physically transparent position-dependent local dielectric permittivity near a surface or an interface, $r_{0}$ needs to be as small as possible, but it should not be smaller than the decay length $\xi$ of the nonlocal susceptibility. In terms of these properly averaged quantities, Eqs. (1) and (2) can simplify ${ }^{17}$ to

$$
\overline{\bar{D}}(z)=\varepsilon(z) \overline{\bar{E}}(z) \text { and } \overline{\bar{P}}(z)=\chi(z) \overline{\bar{E}}(z),
$$

where the double bars indicate the averages taken over the $x y$ plane and the $z$ direction. Naturally, Eq. (7) defines our local dielectric permittivity and susceptibility. In practical applications, considering that $\overline{\bar{D}}(z)$ is continuous, it is convenient to express the local permittivity in terms of the polarization $\overline{\bar{P}}(z)$, i.e.,

$$
\varepsilon(z)=1+4 \pi \chi(z)=1+\frac{4 \pi \overline{\bar{P}}(z)}{D-4 \pi \overline{\bar{P}}(z)},
$$

where $D$ is the external field of a finite system. For an extended system, $D=E_{\mathrm{av}}+4 \pi P$, where $E_{\mathrm{av}}$ corresponds to the average self-consistent electric field, and $P$ is the fieldinduced macroscopic polarization in the supercell. The latter can be determined by Berry-phase method. ${ }^{18}$ Thus, Eq. (8) together with Eq. (5) establish a direct link between the induced charge density and the local permittivity. By calculating $\bar{\rho}_{\text {ind }}(z)$ with the atoms fixed or relaxed in the finite electric field, we can obtain the corresponding optical and static permittivity profiles.

In addition to the above, Giustino and Pasquarello 9 also analyzed the local permittivity in terms of discrete effective electronic and ionic polarizabilities. For the electron charge density, decomposition into maximally localized Wannier functions $^{12}$ provides a discrete representation of the local 
contributions. They define the effective electronic polarizability $\alpha_{\text {elec }}^{(n)}$ for each Wannier function by relating its induced dipole to the electric field at its center $z_{n}$

$$
e\left(z_{n}^{E}-z_{n}^{E=0}\right) \equiv \alpha_{\text {elec }}^{(n)} \overline{\bar{E}}\left(z_{n}\right) .
$$

This definition relates the variation in the dielectric response to the specific features of the local bonding arrangement. In analogy with $\alpha_{\text {elec }}^{(n)}$, they also define the effective ionic polarizability as

$$
\sum_{\beta} Z_{I, z \beta}^{D}\left(R_{I \beta}^{E}-R_{I \beta}^{E=0}\right) \equiv \alpha_{\text {ion }}^{(I)} \overline{\bar{E}}\left(R_{I z}\right),
$$

where $R_{I \beta}$ and $Z_{I, z \beta}^{D}$ represent the coordinate and the dynamical charge of the ion $I$. The dynamical charge is determined from the linear relation between the force on an atom $F_{I \beta}$ and the effective local field $\overline{\bar{E}}_{z}$,

$$
Z_{I, z \beta}^{D} \equiv \frac{\partial F_{I \beta}}{\partial \overline{\bar{E}}\left(R_{I z}\right)} .
$$

In our calculations, based on $\alpha_{\text {ion }}^{(I)}$, we also define an effective layer ionic polarizability by

$$
\alpha_{\text {layer }} \equiv \frac{1}{S} \sum_{I} \alpha_{\text {ion }}^{(I)},
$$

where $S$ is the transverse area of the simulation cell and the summation is over all atoms belonging to the same layer. Equation (12) is well justified because $\overline{\bar{E}}$ is almost the same for all the atoms belonging to the same layer.

In Eqs. (9) and (10), the $\alpha_{\text {elec }}^{(n)}$ and $\alpha_{\text {ion }}^{(I)}$ describe the extent of electronic and ionic contributions to the local polarization around the center of the Wannier function or the ion, respectively. However, because of nonlocality, the contributions of nearby Wannier functions and ions should also be considered when evaluating the local permittivity at a given position, depending on the decay length of the nonlocal microscopic susceptibility. For a layered structure, the neighboring layers should also be included.

\section{METHODOLOGY AND CALCULATIONS}

\section{A. Evaluation of the decay length of the nonlocal susceptibility}

As seen in Sec. II, the local dielectric permittivity should be obtained from a suitable averaging procedure. Only when the average is taken over a distance $r_{0}$ that is larger than the decay length $\xi$ of the microscopic susceptibility, can the derived permittivity profiles be considered local and physically meaningful. The determination of $\xi$ is thus the key for ascertaining the locality of the derived permittivity profile. The microscopic susceptibility $\chi\left(\mathbf{r}, \mathbf{r}^{\prime}\right)$ can be equivalently defined as the linear response of the charge density $\delta \rho(\mathbf{r})$ to an external potential perturbation $\delta V_{\text {ext }}\left(\mathbf{r}^{\prime}\right)$, i.e.,

$$
\delta \rho(\mathbf{r})=\int \chi\left(\mathbf{r}, \mathbf{r}^{\prime}\right) \delta V_{\text {ext }}\left(\mathbf{r}^{\prime}\right) d \mathbf{r}^{\prime},
$$

which can be formally written in term of a functional derivative

$$
\chi\left(\mathbf{r}, \mathbf{r}^{\prime}\right)=\frac{\delta \rho(\mathbf{r})}{\delta V_{\mathrm{ext}}\left(\mathbf{r}^{\prime}\right)}
$$

From this definition, for a given very localized external potential disturbance at position $\mathbf{r}^{\prime}$, the corresponding induced charge density $\delta \rho$ at $\mathbf{r}$ characterizes the nonlocality of the microscopic susceptibility $\chi\left(\mathbf{r}, \mathbf{r}^{\prime}\right)$. The spatial decay of $\delta \rho$ can then be used to obtain the decay length $\xi$ of $\chi\left(\mathbf{r}, \mathbf{r}^{\prime}\right)$. We, therefore, evaluate $\xi$ directly from the decay of the induced charge density by displacing an ion by a very small amount $\Delta u$ from its equilibrium position in a supercell, resulting in a very localized dipolar external potential that is quickly screened by the electrons. The supercell is chosen large enough so that the induced charge density does not overlap between the cells.

In general, the induced charge-density profile $\delta \rho(\mathbf{r})$ in an insulator should have a small, but slowly decaying tail, corresponding to the screening by the finite dielectric constant. However, in density-functional theory (DFT) the screening is rather rapid, ${ }^{19}$ both when using local-density approximation (LDA) or generalized gradient approximation (GGA). In our supercells we observe a rather rapid decay, sometimes with small oscillations, which still decay to zero before reaching the supercell boundary. In order to provide a physically meaningful lower bound of nonlocality of $\chi\left(\mathbf{r}, \mathbf{r}^{\prime}\right)$, we define $\xi$ due to a particular atom as a spatial extent of the region containing $95 \%$ of $|\delta \rho|$, viz.

$$
\int_{z_{0}-\xi / 2}^{z_{0}+\xi / 2}|\delta \rho(z)| d z / \int_{L}|\delta \rho(z)| d z=p=95 \%,
$$

where $z_{0}$ is the $z$ coordinate of the atom being displaced and $L$ is the supercell size along $z$.

In fact, the above method for obtaining $\xi$ corresponds to calculating the broadening width of a dynamical charge ${ }^{20}$ for a given atom along a certain direction. Within a linear regime, the dynamical charge and its broadening width (i.e., $\xi$ ) show little dependence on the magnitude of $\Delta u$ for the same atom at different $\mathbf{r}$, even for a nonuniform material. This is shown in Fig. 1, where the $\xi$ is calculated by displacing atoms at different positions with varying $\Delta u$ in an isolated (001) $\alpha-\mathrm{Al}_{2} \mathrm{O}_{3}$ slab. In principle, the smaller $\Delta u$ correspond to a more localized disturbance and should yield a more accurate $\xi$. Nevertheless, for consistency with permittivity calculations, we choose $\Delta u$ to be the maximum atomic displacement caused by the electric field used for deducing dielectric functions. However, the dynamical charge and its broadening width are different for atoms with different numbers of valence electrons and ionicities. For example, by displacing $\mathrm{Al}$ and $\mathrm{O}$ atoms we find $\xi=8.9$ and 4.3 a.u., respectively. To ensure the locality of the dielectric permittivity everywhere in the slab considered in the next sections, we choose the maximum $\xi$ of all atoms in our averaging procedure.

\section{B. Nanoscale averaging model}

The averaging procedure can be explicitly defined by using a real normalized weight function $f$, which satisfies 


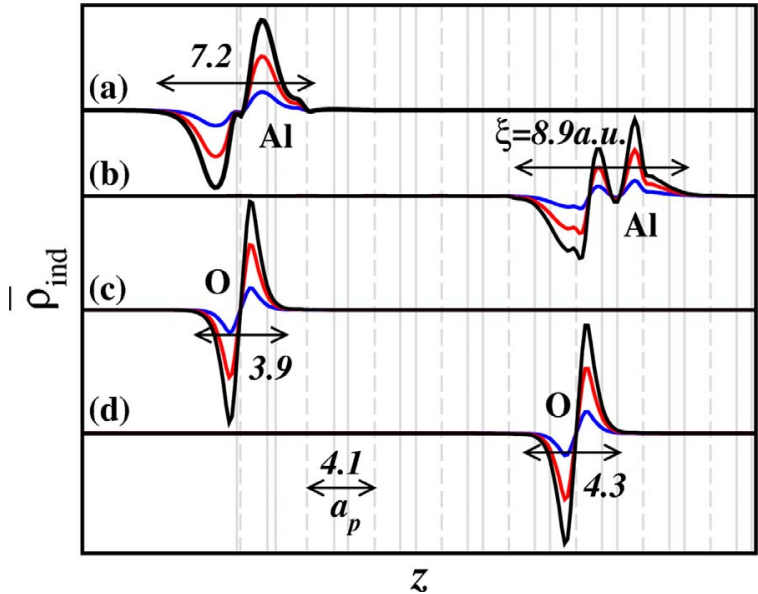

FIG. 1. (Color online) Planar average of the atomicdisplacement-induced charge densities along $z$ in a (001) $\alpha-\mathrm{Al}_{2} \mathrm{O}_{3}$ slab. (a)-(d) correspond to the density profiles induced by, respectively, moving the surface $\mathrm{Al}$ layer, the middle $\mathrm{Al}$ layer, the surface $\mathrm{O}$ layer, and the middle $\mathrm{O}$ layer of the slab. The corresponding layer in (a)-(d) is moved by three different $\Delta u: 0.001,0.003$ and 005 a.u., denoted by curves with highest peaks, medium peaks, and lowest peaks, respectively. Vertical gray lines indicate the positions of $\mathrm{O}$ (dashed line) and Al (solid line) atomic layers in the slab. The periodic interlayer distance in the middle of the slab is indicated as $a_{p}$.

$$
\int f\left(z-z^{\prime}\right) d z^{\prime}=1 ; \quad f\left(z-z^{\prime}\right)=0, \quad\left|z-z^{\prime}\right|>r_{0} .
$$

For the electric field we then have

$$
\overline{\bar{E}}(z)=\int \bar{f}\left(z-z^{\prime}\right) \bar{E}\left(z^{\prime}\right) d z^{\prime} .
$$

Equation (17) shows that $\overline{\bar{E}}(z)$ depends on the choice of the $f$. The electric displacement is invariant after any averaging because of Eq. (6), i.e., $\overline{\bar{D}}(z)=\bar{D}(z)$. As a result, the dielectric permittivity derived from Eq. (7) is not unique and depends on the choice of the weight function $f$.

In actual calculations two kinds of weight functions are commonly used. One is a rectangular filter ${ }^{21}$

$$
f_{l}(z)=\frac{1}{l} \Theta\left(\frac{l}{2}-|z|\right),
$$

where $\Theta$ is a unit-step function and $l$ is the layer periodicity. The $f_{l}$ is best suited for a superlattice composed of undistorted crystalline layers. However, for a system with varying planar distances, this method is not efficient in filtering oscillations that are due to different layer spacings. The other commonly used function is the Gaussian broadening kernel ${ }^{9}$

$$
f_{\sigma}(z)=\frac{1}{\sqrt{2 \pi} \sigma} e^{-z^{2} / 2 \sigma^{2}},
$$

which is continuous at any $z$ and suitable for disordered systems with only short-range order. However, since $f_{\sigma}$ does not explicitly include the periodic lattice spacing information, it is less efficient in flattening the permittivity profile in the bulklike region away from the interface.

In this work, we are mainly dealing with crystalline slabs, which usually contain uniformly spaced atomic planes in the regions far away from surfaces or interfaces, while near surfaces or interfaces this spacing becomes nonuniform. The slab must be thick enough to recover bulk permittivity in its middle region, but we are, in particular, interested in the widths of the surface or interface regions. Efficient averaging, which would quickly lead to a flat permittivity profile in the middle of the slab while preserving the main features in the surface and interface regions, would thus provide maximum physical insight and minimize the computational expense. We thus choose $f$ as a convolution of the two broadening functions

$$
f_{l \sigma}(z)=\int f_{l}\left(z-z^{\prime}\right) f_{\sigma}\left(z^{\prime}-z^{\prime \prime}\right) d z^{\prime} d z^{\prime \prime} .
$$

To ensure the locality of the extracted dielectric functions, we further require that the broadening width $r_{0}$ of this convolution satisfies

$$
r_{0} \geq \xi
$$

In our calculations, $l$ is set to the lattice spacing of the bulk region away from the surface or interface and $\sigma$ is then chosen so that Eq. (21) is satisfied. However, similarly to the decay length of the induced charge density, there is also some arbitrariness in defining the broadening width $r_{0}$ of this convolution. In order to be consistent with the numerical definition of $\xi$, we define $r_{0}$ through Eq. (15), with $|\delta \rho(z)|$ replaced by $f_{l \sigma}(z)$. To confirm that $f_{l \sigma}(z)$ satisfies Eq. (21) for the particular choices of $l$ and $\sigma$, we also validate this condition visually by plotting the normalized $|\delta \rho|$ and $f_{l \sigma}$ in the same figure and centering at the same position. This procedure provides a robust lower bound of $\sigma$ that satisfies Eq. (21). The actual averaging with $f_{l \sigma}$ is easily carried out in reciprocal space by multiplying each Fourier coefficient with the weight

$$
f_{G}=\frac{\sin (G l / 2)}{G l / 2} e^{-G^{2} \sigma^{2} / 2} .
$$

\section{Calculations}

The calculations were performed using density-functional theory and ultrasoft pseudopotentials, as implemented in the QUANTUM-ESPRESSO package. ${ }^{22}$ For alumina and iPP slabs and their interfaces, we use the exchange and correlation functional in the generalized gradient approximation. For the $\mathrm{PbTiO}_{3}$ slab and $\mathrm{PbTiO}_{3} / \mathrm{iPP}$ interface the local-density approximation is used because the GGA catastrophically overestimates both equilibrium volume and $c / a$ of $\mathrm{PbTiO}_{3} .{ }^{23}$ The wave functions and the augmented charge density are represented by plane-wave basis sets with energy cutoffs of 30 and 300 Ry, respectively. All atoms in the supercell are relaxed until the atomic forces are less than $3 \mathrm{meV} / \AA$. Our calculated bulk dielectric constants and lattice parameters of $\alpha-\mathrm{Al}_{2} \mathrm{O}_{3}$, tetragonal $\mathrm{PbTiO}_{3}$, and monoclinic iPP are in ex- 
TABLE I. Bulk dielectric constants and lattice parameters for monoclinic iPP, hexagonal $\mathrm{Al}_{2} \mathrm{O}_{3}$, and tetragonal $\mathrm{PbTiO}_{3}$. GGA was used for iPP and $\mathrm{Al}_{2} \mathrm{O}_{3}$, and $\mathrm{LDA}$ for $\mathrm{PbTiO}_{3}$. See text.

\begin{tabular}{|c|c|c|c|c|c|c|}
\hline & \multicolumn{2}{|l|}{ iPP } & \multicolumn{2}{|c|}{$\mathrm{Al}_{2} \mathrm{O}_{3}$} & \multicolumn{2}{|c|}{$\mathrm{PbTiO}_{3}$} \\
\hline & Expt. (Ref. 24) & Calc. & Expt. (Ref. 25) & Calc. & Expt. (Ref. 26) & Calc. \\
\hline$a(\AA)$ & 6.65 & 6.73 & 4.76 & 4.77 & 3.91 & 3.86 \\
\hline$b$ & 20.96 & 21.13 & & & & \\
\hline$c$ & 6.51 & 6.53 & 12.99 & 13.04 & 4.14 & 4.01 \\
\hline$\alpha\left(^{\circ}\right)$ & 99.30 & 99.17 & & & & \\
\hline$\epsilon(\infty)$ & 2.30 & 2.25 & 3.25 & 3.22 & 7.13 & 7.24 \\
\hline$\epsilon(0)$ & $2.20-3.35$ & 2.30 & $9.4,11.6$ & $9.8,12.2$ & $32-35$ & 36.4 \\
\hline
\end{tabular}

cellent agreement with experimental ones (see Table I).

The slab structures used to investigate the oxide surfaces and oxide/polymer interfaces are constructed in an orthorhombic supercell, with periodically repeated slabs or stacked films separated by a $20 \AA$ vacuum region along the $z$ axis. We derive the optical and static local dielectric profiles from Eq. (7) as described in Sec. II. The induced charge densities appearing in Eqs. (4) and (5) are calculated as differences between the total charge densities corresponding to two oppositely directed macroscopic electric fields of \pm 0.001 a.u.. The finite electric field can be applied by either using the dipole correction method ${ }^{27,28}$ or the method based on Berry-phase theory of polarization. ${ }^{29,30}$ Here we choose the former one because it removes well the artificial periodic image interactions due to the supercell approach, ${ }^{31}$ while avoiding the convergence issues related to the latter method. $^{32}$

\section{RESULTS AND DISCUSSION}

In this section we first consider metal-oxide slabs in vacuum, which enable analysis of permittivity profiles near surfaces for both paraelectric $\left(\alpha-\mathrm{Al}_{2} \mathrm{O}_{3}\right)$ and ferroelectric $\left(\mathrm{PbTiO}_{3}\right)$ slabs. We also examine a polypropylene slab to establish its permittivity profile. In the last subsection we analyze the metal-oxide/polymer interfaces, and compare their dielectric profiles to those of the constituent materials. The computed profiles can also be used in mesoscopic modeling of dielectric properties of nanocomposites.

\section{A. Metal-oxide slabs}

\section{1. $\alpha-\mathrm{Al}_{2} \mathrm{O}_{3}$ slabs}

For alumina, we consider two surfaces, the single-Olayer-terminated (110) and the single-Al-layer-terminated (001) surfaces. From bond-counting rules of Harrison, ${ }^{33}$ both surfaces should be chemically stable and charge neutral. The latter surface has been observed in the experiment. ${ }^{34}$ The relaxed surface structures in our calculations agree very well with other $a b$ initio calculations. ${ }^{35}$

For both kinds of slabs, our calculated $\xi$ 's [5.9 a.u. for (110) slabs and 8.9 a.u. for (001) slabs] are found to be larger than their layer stacking periodicities [4.5 a.u. for (110) slabs and 4.1 a.u. for (001) slabs]. To obtain the local dielectric permittivities, we choose the averaging weight functions, as described in Sec. III B, with $l=4.5$ a.u. and $\sigma=1.2$ $\left(r_{0}=6.8\right.$ a.u.) for the (110) slabs, and $l=4.1$ a.u. and $\sigma$ $=3.0\left(r_{0}=12.7\right.$ a.u. $)$ for $(001)$ slabs. We have confirmed that the weight functions with these parameter values are broader than their corresponding induced charge-density profiles by plotting them together in the same figure (see Sec. III B).

To investigate the size dependence of the slab permittivities, we calculated the optical and static permittivity profiles for both kinds of slabs with sizes of 6-24 atomic layers. Figure 2(a) shows these profiles for alumina(110) slabs. For slabs thicker than 12 atomic layers ( $\sim 26$ a.u.), the optical permittivity profile in the middle of the slab is flat and matches the corresponding bulk value very well (within $1 \%$ ). For the static permittivity, a flat bulklike permittivity is obtained for 18 layers and beyond. In the surface regions of

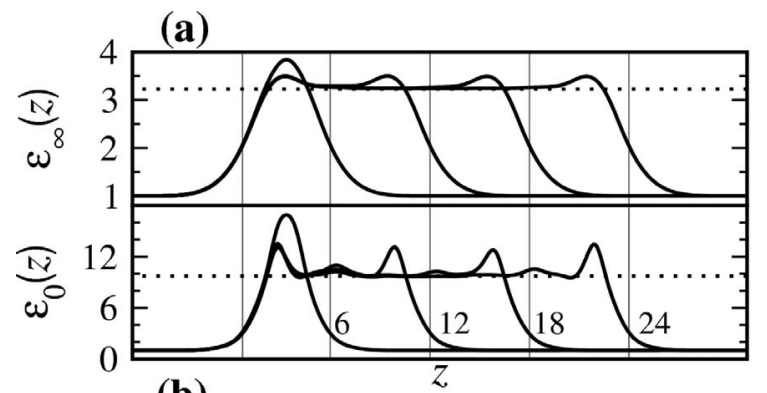

(b)

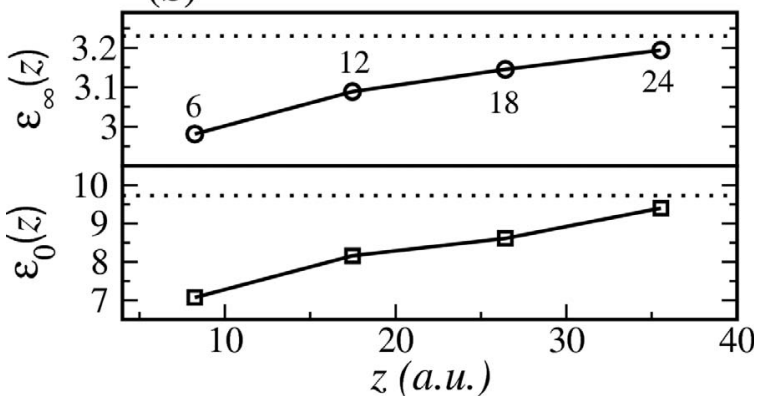

FIG. 2. (a) Dielectric permittivity profiles of $\alpha-\mathrm{Al}_{2} \mathrm{O}_{3}(110)$ slabs along the (110) direction. Vertical lines represent the edge planes of the corresponding slabs. All slabs are aligned at the leftmost surface. (b) Overall effective dielectric permittivities, both high frequency and static, of the four $\alpha-\mathrm{Al}_{2} \mathrm{O}_{3}(110)$ slabs in (a). The horizontal dotted lines show the corresponding bulk permittivities. The number of layers in the slabs is indicated in the figure. 


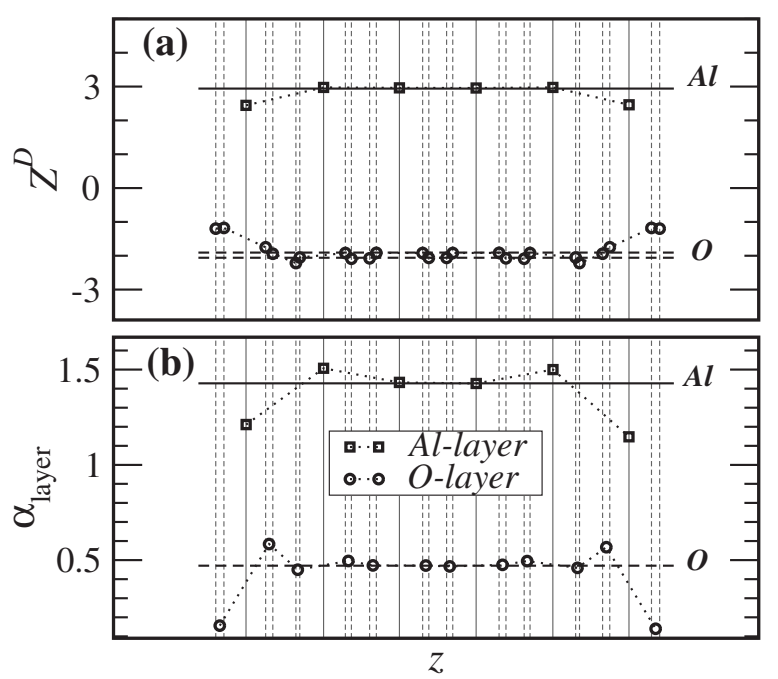

FIG. 3. (a) Dynamical charges and (b) effective ionic layer polarizability for an 18-layer alumina(110) slab as same as the one used in Fig. 2. The two horizontal lines in (a) correspond to the dynamical charges of $\mathrm{Al}$ (solid) and $\mathrm{O}$ (dashed), respectively, in bulk. The two horizontal lines in (b) are only shown for the convenience of comparison between surface and interior. Vertical lines denote the positions of $\mathrm{Al}$ (solid) and $\mathrm{O}$ (dashed) planes along $z$.

these slabs, both the optical and static permittivity profiles have the same shape and are independent of slab thickness. The permittivities increase near the surface and then decrease quickly when approaching the outermost surface layer. As the slab size goes below 18 (or 12) atomic layers, the static (or optical) dielectric permittivity in the slab interior is affected by the surfaces. This coupling becomes stronger as the slab size decreases. As a result, the bulklike dielectric behavior can no longer be recovered in the mid region of such thin slab. For instance, in the middle of a six-layer (110) slab, both the static and optical permittivities are much larger than the corresponding bulk ones. The results for alumina(001) slabs are similar and not shown.

Overall, the combined effects of the two surface regions lead to a reduced effective dielectric permittivity for a thin slab, when compared to the bulk value. This can be seen in Fig. 2(b), where the slab thickness is defined as the distance between the two outermost surface layers. However, the decrease in the overall dielectric permittivity becomes smaller as the slab thickness increases. For a (110) slab with 24 atomic layers (18.7 $\AA$ ), the effective permittivity is already very close (within 5\%) to the bulk value. This implies that alumina slabs with thicknesses greater than a few nanometers can have bulklike dielectric properties. Similar dependence of permittivity on the slab thickness has also been found in finite $\mathrm{Si}$ systems. ${ }^{9,36}$

Figure 2 also shows that the ionic contribution dominates the static dielectric permittivity across the entire slab. To understand in more detail how the ionic contributions determine the surface dielectric properties, we calculated the dynamical charges $Z^{D}$ and effective ionic layer polarizabilities, according to Eqs. (11) and (12), for an 18-layer alumina(110) slab. Figure 3(a) indicates that the effective dynamical charges in the middle of slab agree well with the correspond- ing dynamical charges in the bulk. In the surface region, both $\mathrm{Al}$ and $\mathrm{O}$ have reduced effective charges in their corresponding outermost layers.

From the calculated $Z^{D}$, we obtain the effective ionic layer polarizability profile, shown in Fig. 3(b). We observe that the $\mathrm{Al}$ layers are much more polarizable than the $\mathrm{O}$ layers. The ionic layer polarizability profiles of both $\mathrm{Al}$ and $\mathrm{O}$ layers display features similar to the static permittivity profile. Clearly, the enhancement of the local dielectric permittivity close to the surface is due to the second outermost Al layer and second outermost $\mathrm{O}$ layer, which are more polarizable than the corresponding layers in the slab interior. When approaching the outermost surface layer, the local static permittivity decreases quickly because of the much lower polarizabilities of the outermost layers.

\section{2. (001) $\mathrm{PbTiO}_{3}$ slabs}

In the ionic limit, a (001)-oriented $\mathrm{PbTiO}_{3}$ slab can be considered as an alternating stack of neutral $\mathrm{PbO}$ and $\mathrm{TiO}_{2}$ layers. We consider a (001) slab with 13 atomic layers. The calculated decay length of $\chi\left(z, z^{\prime}\right)$ for this slab is about 8.2 a.u. (about three atomic layers), confirming that this slab is thick enough to avoid the surface-surface interactions.

In a zero-field calculation, open-circuit boundary conditions are adopted. However, under these conditions any ferroelectric slab with a net polarization perpendicular to the surface relaxes into a paraelectric nonpolar structure. This is because the net polarization leads to surface charges that give rise to a huge depolarization field inside the slab. The depolarization field contributes to a positive electrostatic energy, which is large enough to completely destabilize the bulk ferroelectric state. ${ }^{28}$ For instance, a 13-layer $\mathrm{TiO}_{2}$-terminated free-standing $\mathrm{PbTiO}_{3}$ slab, which was obtained by truncating a relaxed bulk structure, relaxes into an anticentrosymmetric layered domain structure and becomes nonpolar as a whole. From the outermost surface to the innermost layer, the relative displacement between the metal and the oxygen atoms (rumpling) oscillates in sign from layer to layer, and its amplitude decreases rapidly to zero, as shown in Fig. 4.

To preserve ferroelectricity in such a slab, the depolarization field must be screened. In real systems, this screening may be provided by free charges from metallic electrodes or by compensating surface layers, which may include adsorbates or charged defects such as cation or oxygen vacancies. In our calculations, we use $\mathrm{Nb}$-doped and Sc-doped surfaces to compensate the depolarization field. This chargecompensating method has already been employed to stabilize domain walls in other $\mathrm{PbTiO}_{3}$ calculations. ${ }^{37}$ Specifically, in the $2 \times 2$ 13-layer $\mathrm{TiO}_{2}$-terminated $\mathrm{PbTiO}_{3}$ slab, we replace three $\mathrm{Ti}$ atoms by $\mathrm{Nb}$ on one surface, and three $\mathrm{Ti}$ atoms by Sc on the other surface, creating deltalike compensating surface charge densities of about \pm 0.0141 e/a.u. ${ }^{2}$, which are very close to those induced by spontaneous bulk polarization of $\mathrm{PbTiO}_{3}\left(0.0134 \mathrm{e} / \mathrm{a} . \mathrm{u}^{2}\right)$. After relaxation, this slab now remains in a single-domain ferroelectric state (see Fig. 4), with slightly increased layer rumpling parameters and $c / a$ ratio in the middle of the slab. 


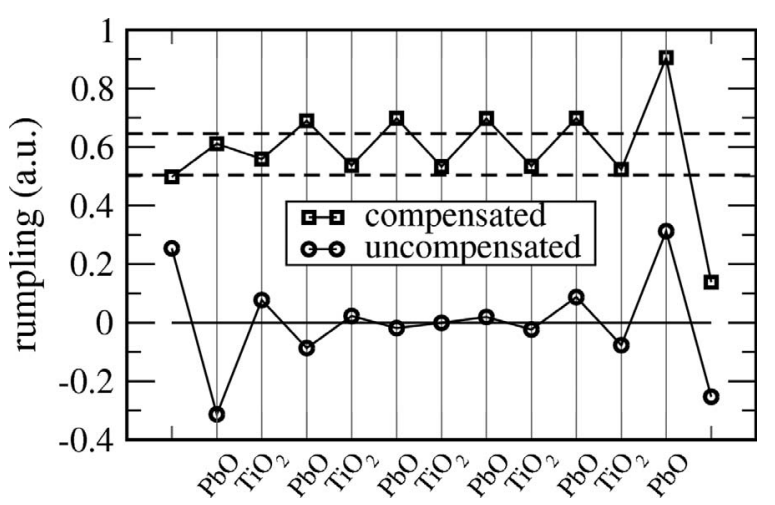

FIG. 4. Layer rumpling (the relative displacement of the oxygen atom with respect to the metal atom along $c$ ) for free standing $\mathrm{PbTiO}_{3}$ slabs with (square) and without (circle) surface charge compensation. Horizontal dashed lines represent the corresponding rumpling of the $\mathrm{TiO}_{2}$ and $\mathrm{PbO}$ layers in the bulk.

The local dielectric permittivity profiles for this compensated slab are shown in Fig. 5. In the averaging model we use $l=7.63$ a.u., which is equal to the lattice spacing in the slab interior, and $\sigma=2.5$. The resulting weight function $\left(r_{0}\right.$ $\sim 13.0$ a.u.) is broader than the induced charge-density profile $(\xi \sim 8.2$ a.u.). For these choices we observe that in the slab interior both the optical and static dielectric permittivity profiles are flat and slightly lower than the corresponding bulk values. This permittivity reduction originates from the slightly over-compensating surface charges. As a response to the remanent surface charges, the lattice constant $c$ in the slab mid region increases by about $1 \%$. For a strained bulk with this enlarged $c$ and $a$ fixed at its theoretical bulk value, we find that the optical and static permittivities become 7.13 and 33.95 , respectively, matching very well the corresponding permittivities in the slab interior.

In the surface regions, similar to Si slabs, ${ }^{9}$ the optical dielectric permittivity is reduced due to lower electronic polarizability at the surface. However, the static permittivity is

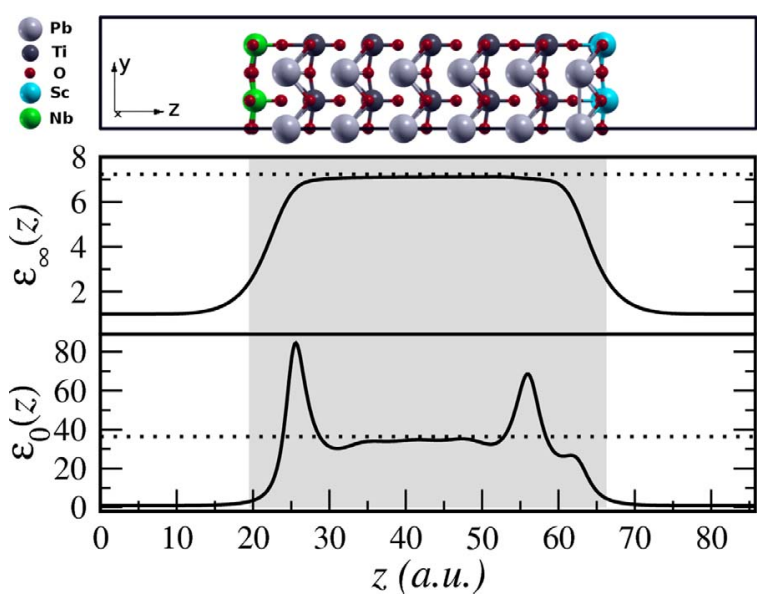

FIG. 5. (Color online) Optical and static dielectric permittivity profiles along $c$ for the $(001) \mathrm{PbTiO}_{3}$ slab with the left side terminated by a $\left(\mathrm{Nb}_{3 / 4} \mathrm{Ti}_{1 / 4}\right) \mathrm{O}_{2}$ surface and the right side terminated by a $\left(\mathrm{Sc}_{3 / 4} \mathrm{Ti}_{1 / 4}\right) \mathrm{O}_{2}$ surface. Horizontal dotted lines represent the bulk permittivities.

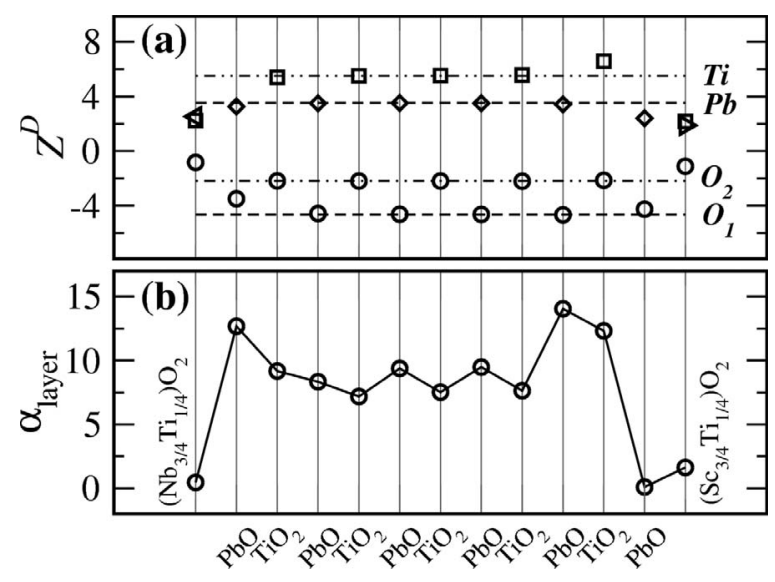

FIG. 6. (a) Dynamical charges and (b) effective ionic layer polarizability profile for the $\mathrm{PbTiO}_{3}$ slab shown in Fig. 5. Vertical lines represent the positions of the atomic layers. In (a), bulk dynamical charges of $\mathrm{Pb}, \mathrm{Ti}$, and $\mathrm{O}$ are indicated by horizontal lines. The triangles at the leftmost and the rightmost layers represent the dynamical charges of $\mathrm{Nb}$ and $\mathrm{Sc}$ atoms, respectively. The dynamical charges of surface $\mathrm{Ti}$ are similar to those of $\mathrm{Nb}$ and $\mathrm{Sc}$, as indicated by the overlapping squares and triangles.

substantially enhanced in the subsurface layers, and differs in shape at the two surfaces. In order to better understand this enhancement, we also calculate the dynamical charges and effective ionic layer polarizabilities for this slab. As shown in Fig. 6(a), the atoms in the two outermost surface layers have smaller dynamical charges, while within the slab interior the effective dynamical charges coincide well with the corresponding ones in the bulk. From the discrete effective ionic layer polarizability profile [Fig. 6(b)], one can observe similar characteristics as in the continuous local permittivity profile in Fig. 5. At the left side of the slab, the outermost surface layer of $\left(\mathrm{Nb}_{3 / 4} \mathrm{Ti}_{1 / 4}\right) \mathrm{O}_{2}$ is much less polarizable, leading to a lower local permittivity. The subsurface layer of $\mathrm{PbO}$ and the third inner surface layer of $\mathrm{TiO}_{2}$ have larger polarizabilities and result in a higher local permittivity in the nearby region. At the other side of the slab, the outermost $\left(\mathrm{Sc}_{3 / 4} \mathrm{Ti}_{1 / 4}\right) \mathrm{O}_{2}$ surface layer is also much less polarizable. Interestingly, the least polarizable layer is the subsurface layer of $\mathrm{PbO}$. The combined effect of these two layers produces a small local permittivity peak at the surface. The more polarizable third and fourth inner surface layers induce a significantly larger local dielectric permittivity peak, as shown in Fig. 5.

\section{B. Polypropylene slabs}

Here we only consider the most common crystal phase of iPP, the $\alpha$ phase. ${ }^{24}$ The unit cell is monoclinic, with the lattice parameters shown in Table I. The cell contains four parallel chains arranged along the $b$ axis. Each chain consists of three $\mathrm{CH}_{2}-\mathrm{CH}\left(\mathrm{CH}_{3}\right)$ monomers and has a threefold helical conformation. In our slab calculations, for computational reasons, an orthorhombic supercell is used instead of the monoclinic one, which only amounts to a change in the angle between $a$ and $c$ from $99.17^{\circ}$ to $90^{\circ}$. In this supercell, the chain conformation and unit chain length are preserved, and 


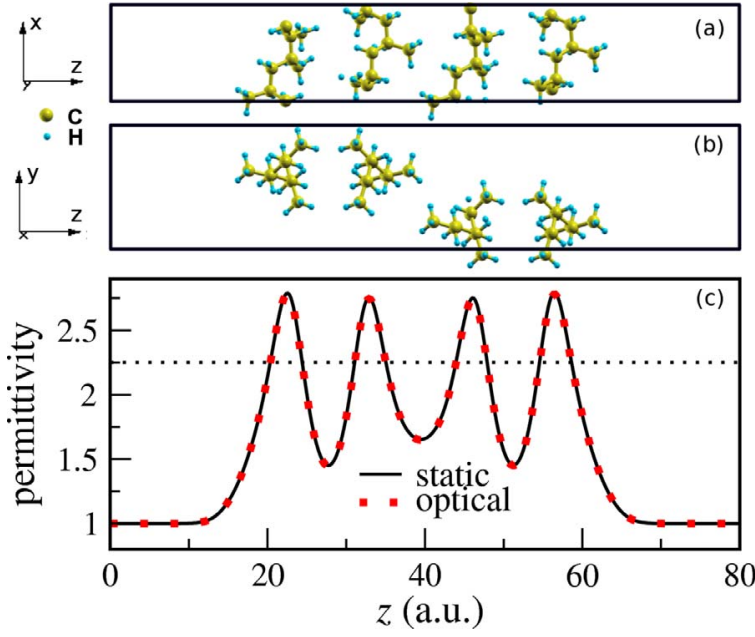

FIG. 7. (Color online) (a) and (b) The atomic structure ( $x z$ and $y z$ sections of the supercell) of the iPP slab with single-methyl surfaces. The double-methyl surface in the text refers to the surface between the surface and the subsurface layers shown here. (c) Optical and static dielectric permittivity profiles for the single-methylterminated slab along $z$.

the change in the dielectric permittivity is less than $3 \%$. It is, therefore, reasonable to assume that the surface dielectric properties are essentially unaffected by this change in the unit cell.

We study the dielectric permittivity profiles across iPP slabs that consist of two bilayers parallel to the $a c$ plane and terminated by either single-methyl or double-methyl groups [see Figs. 7(a) and 7(b)]. The in-plane lattice parameters were chosen equal to the DFT-optimized $a$ and $c$ values in the monoclinic cell. The single-layer thickness along $z$ is 20.0 a.u., which is much larger than the $\xi$ of only 4.9 a.u. along the surface normal. In order to see the permittivity variation across the chain and in the interchain region, and to compare with the iPP/alumina(110) interface profiles below, we choose the same averaging model as the one we used for alumina(110) slabs, i.e., $l=4.5$ a.u. and $\sigma=1.2$, corresponding to a $r_{0}$ of 6.8 a.u.

Figure 7(c) shows the permittivity profiles for the iPP slab with single-methyl surfaces. For the double-methylterminated slabs, the permittivity profiles look very similar and thus are not shown. The figure shows that the lattice contributions to the static dielectric permittivity can be neglected, as in bulk iPP, because of vanishing dynamical charges. The permittivity profile decays very smoothly from the surface to the vacuum, similar to the drop in permittivity from the chain to the interchain region. Inside the slab, since our $r_{0}$ is much less than the interchain distance of iPP (because it was chosen to be consistent with the interface calculations described in the next subsection), the permittivity oscillates from chain to chain around the bulk value. Near the surface, the peak permittivity at the chain increases slightly, while the interchain permittivity decreases with the increase in interchain distance. This implies that lower chain packaging density in iPP would lead to a lower effective permittivity, as would be expected.

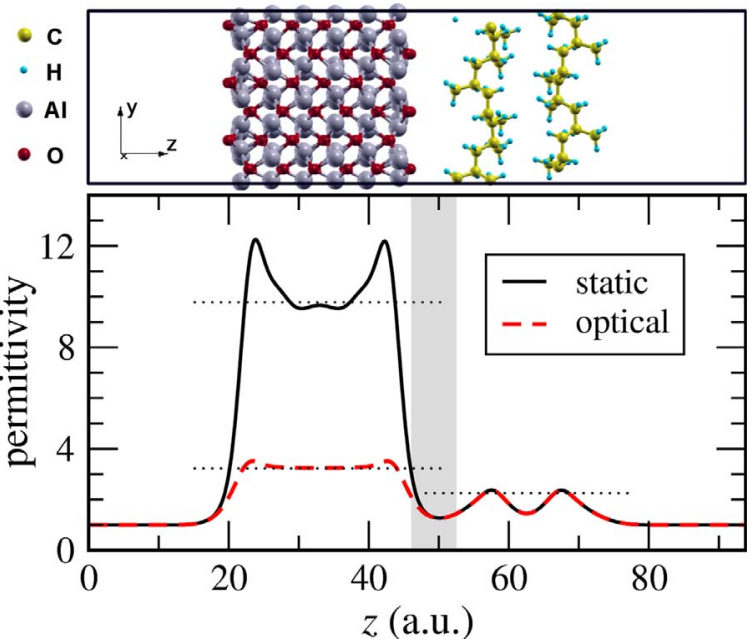

FIG. 8. (Color online) Optical and static dielectric permittivity profiles of the iPP/alumina(110) interface. The $y z$ section of the supercell is shown at the top. The vertical gray bar represents the interface region. The bulk dielectric permittivities corresponding to each slab component are marked by horizontal dotted lines.

\section{C. iPP/metal-oxide interfaces}

The atomic structure of interfaces between polypropylene and metal oxides has not yet been determined. For chemisorbed polypropylene, it could be quite complex and depend on the morphology and quality of the surface of the oxide. In this initial study we thus only examine physisorbed polypropylene, where all the bonds maintain their initial saturation.

\section{1. iPP/alumina interface}

We only consider the single-methyl iPP surface being physisorbed to the oxygen-terminated alumina(110) surface. This interface can be constructed in a moderate size orthorhombic supercell with a lattice mismatch along the chain direction of less than $4 \%$, while other kinds of interfaces would require a much larger supercell to achieve a physically reasonable lattice mismatch.

In the current orthorhombic supercell, two iPP chains are stacked parallel to the $x y$ surface of a 18-layer $\alpha-\mathrm{Al}_{2} \mathrm{O}_{3}(110)$ slab (see Fig. 8). Since iPP polymer is more flexible than the oxide, the in-plane lattice parameters are chosen from the corresponding theoretically relaxed values of bulk $\alpha-\mathrm{Al}_{2} \mathrm{O}_{3}$, namely, $a=\sqrt{3} a_{\mathrm{Al}_{2} \mathrm{O}_{3}}=8.26 \AA, b=c_{\mathrm{Al}_{2} \mathrm{O}_{3}}=13.04 \AA$. The iPP chains are aligned along $y$ (i.e., $b$ ) direction and elongated by $3.2 \%$, whereas along $x$ direction $(a)$ the interchain distance is increased by $26.5 \%$. As a result, the iPP chain density in the surface plane is decreased by $23.4 \%$ relative to the slab in Sec. IV B. Along $z$ direction, as mentioned in Secs. IV A 1 and IV B, the calculated nonlocality lengths for alumina and iPP are 6.8 and 4.9 a.u., respectively. To obtain the local permittivity profiles and be consistent with Secs. IV A 1 and IV B, we use $l=4.5$ a.u. and $\sigma=1.2$, corresponding to $r_{0}$ of 6.8 a.u.

Figure 8(c) shows the optical and static local dielectric permittivity profiles for this interface. In the interior of the alumina slab, both the optical and static permittivities are flat 


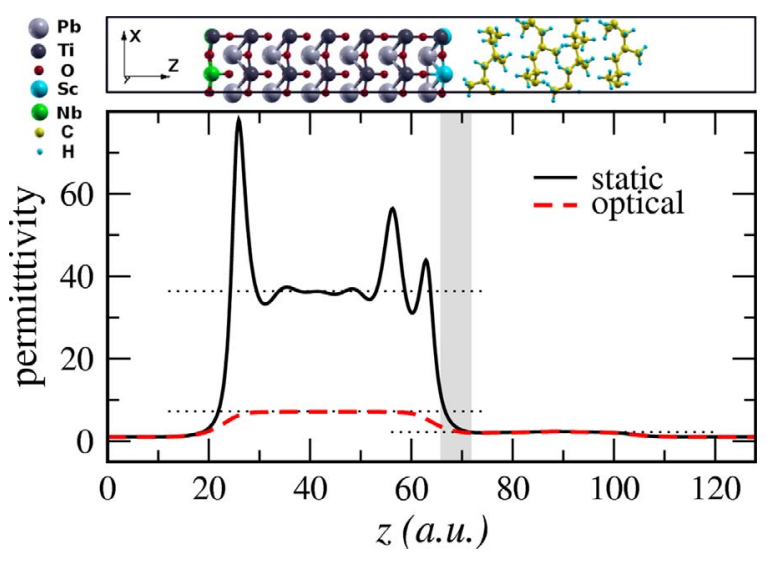

FIG. 9. (Color online) The optical and static dielectric permittivity profiles of the $\mathrm{iPP} / \mathrm{PbTiO}_{3}$ interface. The $x z$ section of the interface structure is shown at the top. The gray vertical bar represents the interface region. The three dotted horizontal lines mark the optical and static permittivity of bulk iPP, and optical and static permittivities of bulk $\mathrm{PbTiO}_{3}$, respectively.

and coincide well with the corresponding bulk values. However, on the iPP side, the permittivity is decreased by about $14 \%$ from its theoretical bulk value. This is because the inplane iPP packing density is reduced by $23.4 \%$. As already seen from Fig. 7 in Sec. IV B, a lower chain density yields lower dielectric permittivity. In the interface region, the interface permittivity profiles of alumina and iPP are quite similar to those of their corresponding isolated surfaces [see Figs. 2(a) and 7(c)]. Proceeding from $\mathrm{Al}_{2} \mathrm{O}_{3}$ to the iPP, the permittivity is enhanced in $\mathrm{Al}_{2} \mathrm{O}_{3}$ close to the interface and then decreases quickly to the smaller dielectric constant of the iPP. The variation in the permittivity across the iPP part of the interface is quite smooth and similar to the permittivity decay from the middle of the chain position to the interchain region. This shows that the interface dielectric effects at the iPP side are insignificant and that the dielectric properties of the interface are dominated by the alumina component. The width of the transition region between alumina and iPP depends thus on the characteristics of the alumina side and is very short, only about 6-7 atomic layers (11 a.u.).

\section{2. $i P P / \mathrm{PbTiO}_{3}$ interface}

We only consider physisorbed polypropylene on surfacecharge-compensated $\mathrm{PbTiO}_{3}$ slab, so that the depolarization field is realistic. To construct a model structure for this interface, we use an orthorhombic supercell containing a 13-layer $\mathrm{PbTiO}_{3}$ slab and a four-layer slab of isotactic polypropylene (iPP). The two slabs are joined together at the $\left(\mathrm{Sc}_{3 / 4} \mathrm{Ti}_{1 / 4}\right)-\mathrm{O}_{2}$ surface, as shown in Fig. 9. In the plane of the interface, we use a $2 \times 2$ unit cell of relaxed $\mathrm{PbTiO}_{3}$, as in the previous subsection. For iPP, one unit of the chain is then matched to

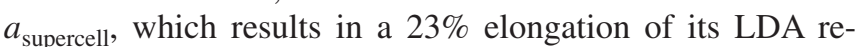
laxed length, and a $16 \%$ elongation of the experimental length. This elongation, although seemingly large, is still much smaller than the experimental breakage elongation of $47 \% .{ }^{38}$ After atomic relaxations, we find that the conformation of the chain remains the same and its dielectric permit- tivity changes very little. Therefore, we expect that this model interface structure can still describe the major dielectric features of a physisorbed polymer/ferroelectric interface.

To obtain the local dielectric permittivity profile, we adopt the same averaging model as for the $\mathrm{PbTiO}_{3}$ slab. We can then easily compare the permittivity profiles of the bare slab with those of the $\mathrm{PbTiO}_{3}$ / iPP slab. The calculated local permittivity profiles of $\mathrm{iPP} / \mathrm{PbTiO}_{3}$ are displayed in Fig. 9. Both optical and static permittivity profiles in $\mathrm{PbTiO}_{3}$ and iPP slab interiors are flat and satisfactorily match the corresponding bulk values. In the interface region, the permittivity decreases rapidly from that of $\mathrm{PbTiO}_{3}$ to the iPP value. The transition region is about 15 a.u. thick and mostly confined to the $\mathrm{PbTiO}_{3}$ side, while at the iPP side the permittivity profile at the interface differs very little from that of bulk iPP. At the $\mathrm{PbTiO}_{3}$ side of the interface, the static permittivity still exhibits two peaks. Compared to the free $\left(\mathrm{Sc}_{3 / 4} \mathrm{Ti}_{1 / 4}\right) \mathrm{O}_{2}$ surface in Fig. 5, the peak closer to the iPP becomes higher and surpasses the bulk value of $\mathrm{PbTiO}_{3}$.

The increased permittivity peak at the $\mathrm{PbTiO}_{3}$ side of the interface originates from the attachment of iPP. A comparison of discrete, effective ion-layer polarizability profiles shows that the two outermost surface layers [i.e., $\left(\mathrm{Sc}_{3 / 4} \mathrm{Ti}_{1 / 4}\right) \mathrm{O}_{2}$ and $\left.\mathrm{PbO}\right]$ of $\mathrm{PbTiO}_{3}$ become much more polarizable when attached to the iPP polymer. By evaluating the individual ionic polarizabilities of all 12 atoms in the $\left(\mathrm{Sc}_{3 / 4} \mathrm{Ti}_{1 / 4}\right) \mathrm{O}_{2}$ layer, we find that the major cause for the increased layer polarizability comes from the Sc atom that is located closest to the side methyl branch of the iPP chain. For this Sc atom, the dynamical charge is increased from 1.90 at the free surface to 2.98 at the interface, and its ionic polarizability correspondingly increases from 66.65 to 269.79. The four $\mathrm{O}$ atoms nearest to this $\mathrm{Sc}$ atom and the nearby Ti atom also have increased ionic polarizabilities and contribute considerably to the increased layer polarizability. From the charge-density difference profile [i.e., $\Delta \rho(z)$ $\left.=\rho_{\mathrm{iPP} / \mathrm{PbTiO}_{3}}-\rho_{\mathrm{iPP}}(z)-\rho_{\mathrm{PbTiO}_{3}}(z)\right]$, we find that the charge density around these six atoms is partially transferred into the middle of the interface. As a result, these ions are screened by fewer electrons and become more polarizable. For the other two Sc and four $\mathrm{O}$ atoms that are farther away from the iPP chain, the charge transfers are very small and their polarizabilities remain almost unchanged. Similarly, for the subsurface $\mathrm{PbO}$ layer, as affected by the $\left(\mathrm{Sc}_{3 / 4} \mathrm{Ti}_{1 / 4}\right) \mathrm{O}_{2}$ layer, the four $\mathrm{Pb}$ atoms become more polarizable and increase the effective ionic polarizability of this layer.

\section{SUMMARY AND CONCLUSIONS}

This work focused on evaluating dielectric permittivity profiles across metal-oxide slabs and metal-oxide polymer interfaces. In order to obtain local permittivity profiles with high resolution, the nonlocality decay length $\xi$ of the microscopic susceptibility tensor is explicitly determined for each system across the slab or interface. The $\xi$ is obtained by introducing small displacements in the atomic positions and evaluating the width of the change in the charge density induced by this disturbance. Using the physical condition that the averaging width of the nonlocal susceptibility must be 
greater than the nonlocality length, we proposed a different procedure for obtaining nanoscale-resolved local dielectric permittivity profile along a given direction. It combines the advantages of the two common averaging models: rectangular filtering and Gaussian kernel. It converges quickly to the bulk value in slabs of only moderate thicknesses, while providing atomic-layer-resolved permittivity profiles even in the presence of significant relaxations and surface structure.

This model was used to obtain dielectric permittivity profiles for alumina, $\mathrm{PbTiO}_{3}$, and iPP slabs, and for physisorbed interfaces of alumina/iPP and $\mathrm{PbTiO}_{3} / \mathrm{iPP}$. The calculations show that the surface and interface effects on dielectric permittivity are very localized (within only a few atomic layers) and that the interface effects are mainly confined to the metal-oxide side.

For polypropylene slabs the permittivity profiles indicate that the surface effects can be neglected and the effective permittivity of the slab depends mostly on the packing density of the chains. Since the effective permittivity is lowered significantly by nanovoids, the free volume in nanocomposites could be a major factor for reducing their effective permittivities.

For both alumina and $\mathrm{PbTiO}_{3}$ slabs, the static dielectric permittivity is enhanced in the subsurface region, but decays rapidly when approaching the outermost layer. The discrete effective ionic layer polarizability profile shows that these effects are due to the more polarizable subsurface layers and less polarizable outermost surface layers.

Overall, due to their very localized surface permittivity effects, alumina slabs and particles with sizes larger than a few nanometers can retain their average macroscopic values of bulk dielectric permittivity.

For the ferroelectric $\mathrm{PbTiO}_{3}$ slab, our results show that its effective dielectric permittivity strongly depends on the elec- trostatic boundary conditions, i.e., the depolarization field. In the slab interior, the permittivity depends on the tetragonality ratio $c / a$, which can be modified by the residual depolarization field. Therefore, the bulklike permittivity can only be obtained after the depolarization field is completely screened, which requires a slab with thickness of more than a few nanometers. However, in a real system, the screening is imperfect. Hence, different surface terminations, defects, molecular or cluster adsorption, etc., will in general affect the effective permittivity of the slab.

While we have only considered physisorbed interfaces, chemically bonded interfaces should have similar properties outside of the immediate bonding region. However, the bonding at the interface will affect surface charges, which would certainly affect the depolarization field. One should, therefore, search for bonding configurations that would enhance the screening of the depolarization field, thereby lowering the minimum sizes of the oxide particles, at which their bulk permittivities are preserved. In general, however, since the range of interface effects extends only over a few atomic layers in charge-compensated slabs, classical mixing laws associated with macroscopic composites should be applicable for modeling the overall dielectric properties of a real polymer/metal-oxide nanocomposite system. Alternatively, a nanoscale computer simulation of the nanocomposite, using the bulk dielectric constants for its constituents, could be employed to arrive at a more detailed description of the dielectric properties, provided that the nanoparticles are larger than a few nanometers.

\section{ACKNOWLEDGMENTS}

This work was supported in part by the Office of Naval Research.
${ }^{1}$ P. Kim, S. C. Jones, P. J. Hotchkiss, J. N. Haddock, B. Kippelen, S. R. Marder, and J. W. Perry, Adv. Mater. 19, 1001 (2007).

${ }^{2}$ R. Schroeder, L. A. Majewski, and M. Grell, Adv. Mater. 17, 1535 (2005)

${ }^{3}$ Y. Cao, P. C. Irwin, and K. Younsi, IEEE Trans. Dielectr. Electr. Insul. 11, 797 (2004).

${ }^{4}$ Y. Bai, Z.-Y. Cheng, V. Bharti, H. S. Xu, and Q. M. Zhang, Appl. Phys. Lett. 76, 3804 (2000).

${ }^{5}$ S. Liang, S. R. Chong, and E. P. Giannelis, Proceedings of the 48th Electronic Components \& Technology Conference, Seattle, WA, 1998, p. 171.

${ }^{6}$ E. Tuncer, Y. V. Serdyuk, and S. Gubanski, IEEE Trans. Dielectr. Electr. Insul. 9, 809 (2002).

${ }^{7}$ D.-H. Yoon, J. Zhang, and B. I. Lee, Mater. Res. Bull. 38, 765 (2003).

${ }^{8}$ Y. Rao, J. Qu, T. Marinis, and C. P. Wong, IEEE Trans. Compon. Packag. Technol. 23, 680 (2000).

${ }^{9}$ F. Giustino and A. Pasquarello, Phys. Rev. B 71, 144104 (2005).

${ }^{10}$ N. Shi and R. Ramprasad, Phys. Rev. B 74, 045318 (2006).

${ }^{11}$ M. Stengel and N. A. Spaldin, Nature (London) 443, 679 (2006).

${ }^{12}$ N. Marzari and D. Vanderbilt, Phys. Rev. B 56, 12847 (1997).
${ }^{13}$ C. Sgiarovello, M. Peressi, and R. Resta, Phys. Rev. B 64, 115202 (2001).

${ }^{14}$ J. P. Calame, J. Appl. Phys. 104, 114108 (2008).

${ }^{15}$ J. Junquera, M. H. Cohen, and K. M. Rabe, J. Phys.: Condens. Matter 19, 213203 (2007).

${ }^{16}$ The microscopic polarization is defined as the transient current induced by an electric field (Refs. 39 and 40).

${ }^{17}$ F. Giustino and A. Pasquarello, Phys. Rev. B 78, 075307 (2008).

${ }^{18}$ R. D. King-Smith and D. Vanderbilt, Phys. Rev. B 47, 1651 (1993).

${ }^{19}$ J. Bernholc, N. O. Lipari, and S. T. Pantelides, Phys. Rev. B 21, 3545 (1980).

${ }^{20}$ P. Ghosez, J.-P. Michenaud, and X. Gonze, Phys. Rev. B 58, 6224 (1998).

${ }^{21}$ L. Colombo, R. Resta, and S. Baroni, Phys. Rev. B 44, 5572 (1991).

${ }^{22}$ P. Giannozzi et al., http://www.quantum-espresso.org.

${ }^{23}$ Z. Wu, R. E. Cohen, and D. J. Singh, Phys. Rev. B 70, 104112 (2004).

${ }^{24}$ G. Natta and P. Corradini, Nuovo Cimento 15, 40 (1960).

${ }^{25}$ W. E. Lee and K. Lagerlof, J. Electron Microsc. Tech. 2, 247 
(1985).

${ }^{26}$ M. D. Fontana, H. Idrissit, G. E. Kugelt, and K. Wojcikt, J. Phys.: Condens. Matter 3, 8695 (1991).

${ }^{27}$ L. Bengtsson, Phys. Rev. B 59, 12301 (1999).

${ }^{28}$ B. Meyer and D. Vanderbilt, Phys. Rev. B 63, 205426 (2001).

${ }^{29}$ P. Umari and A. Pasquarello, Phys. Rev. Lett. 89, 157602 (2002).

${ }^{30}$ I. Souza, J. Íñiguez, and D. Vanderbilt, Phys. Rev. Lett. 89, 117602 (2002).

${ }^{31}$ L. Yu, V. Ranjan, W. Lu, J. Bernholc, and M. Buongiorno Nardelli, Phys. Rev. B 77, 245102 (2008).

${ }^{32}$ P. Umari and A. Pasquarello, Phys. Rev. B 68, 085114 (2003).
${ }^{33}$ W. A. Harrison, J. Vac. Sci. Technol. A 16, 1492 (1979).

${ }^{34}$ J. Ahn and J. W. Rabalais, Surf. Sci. 388, 121 (1997).

${ }^{35}$ E. Wallin, J. M. Andersson, E. P. Münger, V. Chirita, and U. Helmersson, Phys. Rev. B 74, 125409 (2006).

${ }^{36}$ C. Delerue, M. Lannoo, and G. Allan, Phys. Rev. B 68, 115411 (2003).

${ }^{37}$ X. Wu and D. Vanderbilt, Phys. Rev. B 73, 020103(R) (2006).

${ }^{38}$ M. Debolt and R. Robertson, Polym. Eng. Sci. 44, 1800 (2004).

${ }^{39}$ R. M. Martin, Electronic Structure (Cambridge University, Cambridge, England, 2003), Chap. 22.

${ }^{40}$ L. L. Hirst, Rev. Mod. Phys. 69, 607 (1997). 